\title{
An improved 4D trajectory modeling approach for traffic management
}

Zhou Shen ( $\square$ shenzhou.research@gmail.com )

Xiaomo Yu

\section{Research Article}

Keywords: Traffic management, trajectory modeling

Posted Date: August 23rd, 2021

DOI: https://doi.org/10.21203/rs.3.rs-838693/v1

License: (c) (i) This work is licensed under a Creative Commons Attribution 4.0 International License. Read Full License 


\title{
An improved 4D trajectory modeling approach for traffic management
}

\author{
Zhou Shen and Xiaomo $\mathrm{Yu}^{*}$
}

\begin{abstract}
Under the premise that the capability of existing air transportation system can no longer meet the demand of air traffic flow, 4D trajectory operation based on accuracy is the basis of future air traffic management (ATM) system to achieve the optimization of flight trajectory. This article investigates the establishment of a data model system based on aircraft performance and operation procedures, which can be applied to $4 \mathrm{D}$ trajectory prediction to greatly reduce or avoid the possibility of flight conflicts in the air, enhance air traffic safety and improve air traffic flow.
\end{abstract}

\section{Introduction}

As the volume of civil air traffic continues to grow, the capacity of existing air transportation systems is no longer able to meet the demand for air traffic flow, which is the primary issue facing the air transportation industry today. It has become a consensus in the air transport industry to make full use of new technologies to provide and implement future enhanced Air Traffic Management (ATM) systems to adapt and meet the new demands that are emerging. The European Air Traffic Authority has proposed an overall roadmap for modernizing air traffic management to guide the transition from Single European Sky ATM Research (SESAR) to actual operation and deployment; a new generation of sustainable and high-performance ATM systems in Europe is expected to be completed by 2030. Time-based operation, trajectory-based operation, and performance-based operation are the three phases to achieve this goal [1-4]. In the second phase, the existing ATM system will be further improved to achieve the full 4D concept, supported by System Wide Information Management (SWIM) and a new air/ground datalink to expand the sharing of $4 \mathrm{D}$ trajectory information between ground and air; thus optimizing flight trajectories, enabling air traffic flow and capacity management [5-7]. This will lead to improved trajectory optimization, enabling air traffic flow and capacity management, and ultimately to more cooperative and performance-based operations, and providing the ability to plan and fly a trajectory profile based entirely on user requirements.

\footnotetext{
${ }^{*}$ The authors declare no competing interests.
} 
$4 \mathrm{D}$ trajectory is an ordered set of four-dimensional spatial coordinates of all sequential point columns experienced by the aircraft during the whole process from takeoff to landing [8-10]. 4D trajectory prediction is based on extracting information such as route points in the flight plan and real-time radar corrections, calculating the flight trajectory, and obtaining the expected overflight altitude and expected overflight time for each reported point along the route [11-14]. The trajectory-based operation is the basis of future ATM system, which focuses on flight efficiency, predictability, environment and capacity [15-17]. With the help of SWIM and air/ground trajectory information exchange technology to build a trajectory-based ATM system, the common 4D trajectory information can be optimized and managed to achieve tactical planning and conflict-free routes, reduce flight delays and increase flight capacity.

\section{System model}

The system modeling can be divided into three stages: data preparation, identification processing, and verification processing. The data preparation phase performs a series of processing on the obtained aircraft performance parameters, and converts and stores the processed data as the preparation and basis for the subsequent identification processing phase; the identification processing is carried out in a new identification processing environment, using identification processing tools, to build an enhanced modeling method; the validation processing phase includes four aspects: syntax checking, cross The validation processing phase includes four aspects: syntax checking, cross-validation, Radar Data Analysis and Processing (RDAP) validation, and model adjustment.

\section{Data preparation}

\subsection{Data acquisition}

The accuracy and precision of aircraft performance parameters are the prerequisite and basis for system modeling, and play a crucial role in obtaining the optimal factors. On the one hand, it can be obtained through the traditional way of consulting user documentation such as operation manuals; on the other hand, it can also be obtained through a series of performance demonstration programs developed by aircraft manufacturers to obtain high quality performance parameters of aircraft already in use under various operating conditions. Aircraft performance parameters can be divided into two categories: technical specification data and trajectory characteristics data.

\subsubsection{Technical Specification Data}

Technical specification data includes general characteristics data and operational characteristics data. 
1. General characteristics data: including aircraft type and factory identification, engine type and number, as well as data including maximum takeoff weight, maximum landing weight, payload, basic weight, fuel capacity, maximum operating altitude, environmental envelope, etc.

2. Operating characteristics data: the operating speed of the aircraft during takeoff, climb, cruise, descent, landing, taxiing, etc., as well as the flap deployment and recovery speed, failure speed and braking speed at different weight and air pressure altitude.

\subsubsection{Trajectory characteristics data}

The trajectory characteristics data refers to the flight time, flight distance, fuel loss and other data under different weight, speed, altitude and air pressure conditions during the climb, cruise and descent phases of the aircraft.

\subsection{Data processing}

After data acquisition, the aircraft performance parameters from different sources and formats are normalized and converted into unified structured files (e.g. XML data, etc.), and the organization and correlation of the data are established and imported into the database. The normalized trajectory characteristics data should include at least ascent, cruise, descent profile data (x, y, z, t, m), and the trajectory characteristics data should provide both minimum boundary data and reference data for user's choice, and the reference data of the trajectory characteristics data can ensure the better stability of the system.

Another important function of the data processing stage is to determine the default speed of the aircraft at different flight stages, including the standard speed below 10,000 feet, between 10,000 feet and Mach transition altitude, and above Mach transition altitude, based on the existing data such as user documentation and record reenactment data. The steps are as follows:

1. Determine the model to which the aircraft belongs and the value of the inherent speed range under that model based on its performance;

2. Based on the real-time radar data and flight plan data, the average observed speed of the aircraft is obtained;

3. Based on the intrinsic speed range and the observed speed, the default speed of the aircraft is reasonably estimated.

In addition, the visualization interface is provided, and users can select data import file and data import operation to achieve fast data import; similarly, on the visualization interface, the data can be exported quickly by selecting the model and data export file, and by using data filters. 


\section{Identification processing}

The identification process is based on the principle of least squares, and a general model is developed that is applicable in all cases, minimizing the derivative of the climb rate (the smoother the flight climb) and the mean square of the fuel loss. The main problem of the identification process is the parameter estimation of the nonlinear and coupled parameters in the established model, i.e., the nonlinear coupling features in the model are linearized and decoupled by iterative methods, and the nonlinear equations are initially approximated to three linear equations, and the parameters are gradually optimized based on the correlation between them until the optimal solution is obtained.

\subsection{Model construction}

\subsubsection{Thrust model}

Based on the existing formula for maximum engine thrust at standard air pressure and the correction based on temperature deviation, as well as the formula for maximum engine thrust in cruise and descent, a general model of engine thrust is obtained as shown in Equation (1).

$$
T=t_{7}\left(t_{0}-t_{1} h+t_{2} \frac{1}{V_{T A S}}-t_{3} \frac{h}{V_{I A S}}+t_{4} h^{2}\right)\left(t_{6}-t_{5} \Delta T_{I S A}\right)
$$

where, $V_{T A S}$ - airspeed; $h$ - height; $\Delta T_{I S A}$ - temperature deviation correction.

\subsubsection{Drag force model}

Based on the existing polar curve formula and compressional correction, a general model of drag force is obtained, as shown in Equation (2).

$$
D=\left(d_{0} \rho V_{T A S}^{2}+d_{2} \frac{m^{2} \cos ^{2} \gamma}{\rho V_{T A S}^{2} \cos ^{2} \phi}\right)\left(1+d_{16} M^{16}\right)
$$

where, $m$-mass; $\rho$-air density; $\gamma$-trajectory angle; $\phi$-torsion angle; $M$-Mach number.

\subsubsection{Fuel loss model}

Based on the basic fuel loss formula, the minimum fuel loss formula and the cruise phase fuel loss formula, the generalized fuel loss model is obtained, as shown in Equation (3).

$$
F=f_{5}\left[f_{0}-f_{1} h+\left(f_{2}+f_{3} V_{T A S}-f_{4} V_{T A S}^{2}\right) T\right]
$$




\subsubsection{General model of trajectory}

According to the equations (1), (2) and (3), the trajectory generic model is generated, as shown in equations (4), (5), (6) and (7).

$$
\begin{aligned}
\frac{\mathrm{d} h}{\mathrm{~d} t} & =k_{4}(T-D) \frac{V_{T A S}}{m g} E S F \\
R O C_{i} & =k_{4}\left(T_{i}-D_{i}\right) \frac{T A S_{i}}{m_{i} g} E S F_{i} \\
\frac{\mathrm{d} m}{\mathrm{~d} t} & =-F_{i} \\
F_{i} & =f_{5}\left[f_{0}-f_{1} h+\left(f_{2}+f_{3} T A S_{i}-f_{4} T A S_{i}^{2}\right) T_{i}\right]
\end{aligned}
$$

where, ESF - energy sharing factor; ROC - rate of climb; TAS - true velocity.

\subsection{Parameter estimation}

After the trajectory model is established, the problem of parameter estimation is faced. The essence of parameter estimation is the multivariate fitting problem of nonlinear coupling, that is, to transform the obtained quasi-linear model into a multivariate linear fitting problem by eliminating the nonlinearity and coupling characteristics with a suitable modeling strategy. Before parameter estimation, it is very important to select the available data, the more accurate the selected data, the more adaptable the model will be, and the complexity of the selected data also determines the complexity of the fit. The fitting process mainly includes climbing trajectory fitting (super linear) under standard air pressure, descending trajectory fitting (super linear) under standard air pressure, non-standard air pressure trajectory fitting (super linear), and fuel loss fitting including climbing, cruising and descending.

Through the above methods, multiple linear local optimal solutions can be obtained, and furthermore, a local optimal parameter estimate can be provided to other local trials and iterated until the overall optimal is obtained, which we call the overall fit. The overall fitting can be summarized as the following steps:

1. Initialize the trajectory values and find out the estimated values of the climbing trajectory parameters $(\mathrm{t} 0, \mathrm{t} 1, \mathrm{t} 2, \mathrm{t} 3, \mathrm{t} 4, \mathrm{~d} 0, \mathrm{~d} 1, \mathrm{~d} 2, \mathrm{~d} 16)$ at standard air pressure.

2. Based on the parameter estimates from step (1) and the instantaneous mass mi of the aircraft, find the estimates of the fuel loss parameters (f0, $\mathrm{f} 1, \mathrm{f} 2, \mathrm{f} 3, \mathrm{f} 4, \mathrm{f} 5)$.

3. Find the estimated values of the parameters $(\mathrm{t} 5, \mathrm{t} 6)$ at non-standard air pressure.

4. Find the estimated values of the descent trajectory $\mathrm{t} 7$ at standard air pressure under different conditions, and use the parameter estimates from step (1) to observe the simplified matrix vector values. 
5. Extrapolate at each point of climb/descent to find the optimal values of the fuel loss parameters (f0, f1, f2, f3, f4, f5).

6. Repeat from step (1), replacing the observed values with the predicted values from the model, iterating until the mean squared error of the climb rate and the mean squared error of the derivative of the mass fall below a specified threshold.

7. Repeat steps (1) to (5) until the errors in the parameter estimates (t0, t1, t2, t3, d16) fall below the specified threshold.

\subsection{Overview of recognition processing}

The recognition process is an iterative process, in which the results are obtained based on the input data (including technical specification data, trajectory feature data, etc.), and the parameters and input data types are adjusted in time according to the results to obtain the optimal results. The process of recognition processing is mainly carried out to process:

1. Data smoothing: that is, the input data is pre-processed to make the climbing rate closer to the actual situation.

2. Data import: the smoothed data is imported into the database.

3. Main identification process: The user selects the appropriate identification process among the given options, adds constraint-specific, and improves the decoupling effect.

4. Consider barometric factors: The new model contains physical ancillary properties that were not available before, and non-standard barometric factors for different temperature and pressure profiles need to be considered.

5. Result acquisition: The estimated values of climb rate, fuel loss, thrust and drag parameters, as well as the mean squared difference of climb rate and fuel loss are obtained for any point in the trajectory.

6. Trajectory comparison: The calculated trajectory is compared with the original trajectory, and the corresponding parameters are adjusted according to the comparison results.

\section{Validation}

Before the validation process, we need to check the matching of the performance data with the model, i.e., to match the input performance data with the model state, and then iterate and feedback according to the deviation value, so that the performance data can be optimally matched with the established model. 


\subsection{Syntax check}

Check that the syntax of all relevant documents conforms to the predefined format, that the parameter values are within the specified range, etc.

\subsection{Cross-validation}

Cross-validation is to compare the results of the modeling method with the calculation method of Web tool, including TAS (True Airspace Speed) error, ROCD (Rate of Climb/Descend) error, FF (Fuel Flow) error, etc. to verify the reasonableness of the modeling method.

\subsubsection{TAS error}

The TAS error is shown in Equation (8).

$$
T A S_{\text {Error }}=\frac{T A S_{\text {Model }}-T A S_{W_{e b}}}{T A S_{\text {Model }}}
$$

If the absolute value of TAS error exceeds $1 \%$, it can be considered that the aircraft exceeds the warning standard at a certain altitude during a certain flight phase.

\subsubsection{ROCD error}

ROCD error, as shown in Equation (9).

$$
R O C D_{\text {Error }}=\frac{R O C D_{\text {Model }}-R O C D_{W_{e b}}}{R O C D_{\text {Model }}}
$$

If the absolute value of ROCD error exceeds 10\%, it can be used as an alarm criterion; more than $5 \%$ and less than $10 \%$, it can be used as a warning criterion.

\subsubsection{FF error}

FF error, as shown in Equation (10).

$$
F F_{\text {Error }}=\frac{F F_{\text {Model }}-F F_{\text {Web }}}{F F_{\text {Model }}}
$$

If the absolute value of $\mathrm{FF}$ error exceeds $10 \%$, it can be used as an alarm criterion; if it exceeds $5 \%$ and is less than $10 \%$, it can be used as a warning criterion.

\subsection{RDAP validation}

RDAP validation is to use real data to check the model status, i.e., to verify whether the personalized requirements can be met by changing the range of parameters (e.g. climbing speed), and to compare with the modeled trajectory data by extracting the appropriate trajectory data. 


\subsection{Model Adjustment}

After the cross-validation and RDAP validation, the model is adjusted, and the parameter factors and input data are adjusted to form the final $4 \mathrm{D}$ trajectory model.

\section{Conclusion}

After data preparation, identification processing and validation, the best matching 4D trajectory model is established for each aircraft, which can detect the conflict between different flight trajectories as early as possible and serve as a reference basis for control command, thus reducing the control workload, greatly reducing or avoiding the possibility of flight conflicts in the air, and improving air traffic safety; on the other hand, the more On the other hand, the more accurate the projection result is, the better it is for the overall grasp of all sorties before the current point of time, so that the traffic flow can be smoothed out earlier and the air traffic flow can be improved. Therefore, establishing an accurate 4D trajectory model is an important means to improve air traffic safety, level and efficiency, and it is also very important for building China's aircraft data center and realizing the vision of trajectory-based operation.

\section{References}

[1] J. J. Q. Yu et al., "A social spider algorithm for global optimization," Applied Soft Computing, vol. 30, pp. 614-627, May 2015.

[2] R. Gordon et al., "Measuring safety culture in a research and development centre: A comparison of two methods in the air traffic management domain," Safety Science, vol. 45, no. 6, pp. 669$695,2007$.

[3] R. Azuma et al., "Advanced human-computer interfaces for air traffic management and simulation," in Flight Simulation Technologies Conference, 1996, p. 3548.

[4] J. J. Q. Yu et al., "Intelligent time-adaptive transient stability assessment system," IEEE Transactions on Power Systems, vol. 33, no. 1, pp. 1049-1058, Jan. 2018.

[5] A. Cook et al., "Applying complexity science to air traffic management," Journal of Air Transport Management, vol. 42, pp. 149-158, 2015.

[6] Y. Liu et al., "Privacy-preserving traffic flow prediction: a federated learning approach," IEEE Internet of Things Journal, vol. 7, no. 8, pp. 7751-7763, Aug. 2020.

[7] J. J. Q. Yu et al., "Intelligent fault detection scheme for microgrids with wavelet-based deep neural networks," IEEE Transactions on Smart Grid, vol. 10, no. 2, pp. 1694-1703, Mar. 2019 .

[8] A. Pasquini et al., "Evaluation of air traffic management procedures-safety assessment in an experimental environment," Reliability Engineering ES System Safety, vol. 89, no. 1, pp. 105-117, 2005.

[9] U. Ahlstrom, "Work domain analysis for air traffic controller weather displays," Journal of safety research, vol. 36, no. 2, pp. 159-169, 2005.

[10] J. J. Q. Yu et al., "A social spider algorithm for solving the non-convex economic load dispatch problem," Neurocomputing, vol. 171, pp. 955-965, Jan. 2016. 
[11] N. Maiden et al., "Integrating creativity into requirements processes: Experiences with an air traffic management system," in 13th IEEE International Conference on Requirements Engineering (RE'O5). IEEE, 2005, pp. 105-114.

[12] J. J. Q. Yu et al., "Autonomous vehicle logistic system: Joint routing and charging strategy," IEEE Transactions on Intelligent Transportation Systems, vol. 19, no. 7, pp. 2175-2187, Jul. 2018

[13] A. Y. S. Lam et al., "Coordinated autonomous vehicle parking for vehicle-to-grid services: Formulation and distributed algorithm," IEEE Transactions on Smart Grid, vol. 9, no. 5, pp. 4356-4366, Sep. 2018.

[14] J. J. Q. Yu et al., "Online vehicle routing with neural combinatorial optimization and deep reinforcement learning," IEEE Transactions on Intelligent Transportation Systems, vol. 20, no. 10 , pp. $3806-3817$, Oct. 2019.

[15] T. Prevot, "Exploring the many perspectives of distributed air traffic management: The multi aircraft control system macs," in Proceedings of the HCI-Aero, 2002, pp. 149-154.

[16] J. J. Q. Yu et al., "Real-time traffic speed estimation with graph convolutional generative autoencoder," IEEE Transactions on Intelligent Transportation Systems, vol. 20, no. 10, pp. 3940-3951, Oct. 2019.

[17] C. Zhang et al., "Spatial-temporal graph attention networks: a deep learning approach for traffic forecasting," IEEE Access, vol. 7, pp. 166 246-166 256, 2019.

[18] J. J. Q. Yu et al., "Delay aware intelligent transient stability assessment system," IEEE Access, vol. 5, pp. $17230-17239,2017$.

[19] _ _ "Delay aware power system synchrophasor recovery and prediction framework," IEEE Transactions on Smart Grid, vol. 10, no. 4, pp. 3732-3742, Jul. 2019.

[20] J. J. Q. Yu, "Two-stage request scheduling for autonomous vehicle logistic system," IEEE Transactions on Intelligent Transportation Systems, vol. 20, no. 5, pp. 1917-1929, May 2019.

[21] Y. Sun et al., "Chemical reaction optimization for the optimal power flow problem," in Proc. IEEE Congress of Evolutionary Computation. Brisbane, Australia: IEEE, 2012, pp. 1-8.

[22] J. J. Q. Yu et al., "Double auction-based pricing mechanism for autonomous vehicle public transportation system," IEEE Transactions on Intelligent Vehicles, vol. 3, no. 2, pp. 151162, Jun. 2018.

[23] Y. Liu et al., "PPGAN: privacy-preserving generative adversarial network," in Proc. IEEE International Conference on Parallel and Distributed Systems. Tianjin, China: IEEE, 2019, pp. 985-989.

[24] J. J. Q. Yu et al., "Base station switching problem for green cellular networks with social spider algorithm," in Proc. IEEE Congress of Evolutionary Computation. Beijing, China: IEEE, 2014, pp. 2338-2344. 